\title{
The Clinical Characteristics and Magnetic Resonance Analysis of Children with Mild Encephalitis with Reversible Isolated Corpus Callosum
}

\author{
Li Chaoyang ${ }^{1, ~}{ }^{*}$, Yang Jie ${ }^{2}$, Guan Xiaoli ${ }^{2}$, Li Chunhua ${ }^{1}$, Jing Xiaowei ${ }^{1}$, Li Wenling ${ }^{3}$ \\ ${ }^{1}$ Department of Neurology, Shanxi Province Children Hospital, Taiyuan, China \\ ${ }^{2}$ Department of Image Center, Shanxi Province Children Hospital, Taiyuan, China \\ ${ }^{3}$ Department of Scientific Research Department, Shanxi Province Children Hospital, Taiyuan, China
}

Email address:

chaoyangli_bill@126.com (Li Chaoyang)

*Corresponding author

\section{To cite this article:}

Li Chaoyang, Yang Jie, Guan Xiaoli, Li Chunhua, Jing Xiaowei, Li Wenling. The Clinical Characteristics and Magnetic Resonance Analysis of Children With Mild Encephalitis with Reversible Isolated Corpus Callosum. American Journal of Pediatrics.

Vol. 4, No. 2, 2018, pp. 36-40. doi: 10.11648/j.ajp.20180402.14

Received: May 4, 2018; Accepted: June 7, 2018; Published: July 16, 2018

\begin{abstract}
Objective: To summarize the Magnetic Resonance Imaging (MRI) performance and clinical characteristics of children mild encephalitis with reversible isolated corpus callosum pressure (MERS), and analyze the possible etiology. Methods: The clinical data and MRI findings of 11 children with MERS in children's hospital of Shanxi Province from January 2012 to January 2015 were summarized, and then their clinical manifestations and characteristics were analyzed. Results: Among the 11 patients, 5 were male and 6 were female. The age range was 2- 14 years. Clinical manifestations include fever $(7$ cases, 63.6\%), gastrointestinal symptoms ( 9 cases, 81.8\%), drowsiness (5 cases, $45.5 \%)$, convulsions (6 cases, 54.5\%), dizziness ( 2 cases, $18.2 \%)$, cervical positive resistance ( 1 case, $9.1 \%)$, carotid resistance suspicious ( 3 patients [27.3\%]). The appearance of cerebrospinal fluid, white blood cell count, leukocyte classification, protein, glucose and chloride are all normal. MRI showed only abnormal signal in the corpus callosum pressure: a slightly longer T1 signal, a longer T2 signal, a high Diffusion Weighted Imaging (DWI) signal, and a low signal of Apparent Diffusion Coefficient (ADC). Has 4 cases of nuclear magnetic head of abnormal signal in the middle of corpus callosum and ovoid, 6 cases, in the middle and a wide belt of corpus callosum, 1 case was located in the corpus callosum and diffuse wide strip. Eleven children were treated with anti-infective agents and other symptomatic treatment. All of the 11 children were recovered and discharged. The duration of the course is 8 $\mathrm{d}$ and the longest is $21 \mathrm{~d}$. The results of MRI follow - up showed that the callosal pressure lesion disappeared. Conclusion: The clinical manifestations of children's MERS include fever, gastrointestinal symptoms, drowsiness and convulsions, and their characteristic head MRI is a reversible cytotoxic edema with good prognosis.
\end{abstract}

Keywords: Encephalopathy, Splenium of Corpus Callosum, Magnetic Resonance Imaging, Children

\section{Introduction}

The reversibly isolated splenium of corpus callosum (SCC) lesion is very rare, rarely reported in China. So far, according to reports, the etiology of SCC reversible isolated lesions include: mild encephalitis with reversible isolated corpus callosum pressure (MERS) [1], the use of antiepileptic drugs, or the sudden withdrawal of antiepileptic drugs, metabolic disorders (such as vitamin B12 deficiency) [2].
This paper summarizes the clinical data and magnetic resonance (MRI) manifestations of MERS in the hospital from January 2012 to January 2015, and analyzes the possible etiology and pathogenesis of MERS in children.

\section{Materials and Methods}

\subsection{Clinical Data}

There were 11 cases of patients with the reversibility 
isolated corpus callosum from January 2012 to January 2015, including 5 males and 6 females, aged 20 months to 14 years, who were diagnosed in the Children Hospital of Shanxi Province, China.

\subsection{Methods}

The characteristics of the disease were revealed by analyzing the clinical manifestations and cranial magnetic properties of the 11 patients that had been diagnosed.

And GE SIGMA HDE 1.5T MRI was used. All patients were performed the following check: SE sequence shaft a $\mathrm{T} 1 \mathrm{WI}(\mathrm{TR}=1985 \mathrm{~ms}, \mathrm{TE}=25.2 \mathrm{~ms}), \mathrm{T} 2 \mathrm{WI}(\mathrm{TR}=5500 \mathrm{~ms}$, $\mathrm{TE}=135.1 \mathrm{~ms}$ ), sagittal T1WI, coronal T2WI - FLAIR (TR $=9852 \mathrm{~ms}, \mathrm{TE}=116.5 \mathrm{~ms}, \mathrm{TI}=2400 \mathrm{~ms})$ and SE$/ \mathrm{EPI}$ sequence shaft DWI $(\mathrm{TR}=6000, \mathrm{TE}=95 \mathrm{~ms}, \mathrm{~b}=0$ and $\mathrm{b}=$ $1000 \mathrm{~s} /$ was) scan, layer $4 \sim 6 \mathrm{~mm}$ thick.

\subsection{Image Analysis}

The lesion morphology and distribution of the corpus callosum in the axial position: the lesion was in a zonal and oval shape, and the lesion was centered, partial or diffuse. Whether the lesion was isolated or combined with other lesions in the skull; whether the lesion is reversible, whether the clinical symptoms are significantly improved after active treatment, whether the lesion of the corpus callosum of the corpus callosum is disappeared.

\section{The Results}

\subsection{Clinical Data of 11 Children with Clinical Characteristics and Treatment Were Shown in Table 1}

Table 1. Clinical manifestation of 11 patients.

\begin{tabular}{ll}
\hline Symptoms and signs & Number of cases \\
\hline fever & 7 \\
drowsiness & 5 \\
vomiting & 8 \\
diarrhea & 1 \\
neck resistance $(+)$ & 1 \\
neck resistance $( \pm)$ & 3 \\
dizziness & 2 \\
amaurosis fugax & 1 \\
convulsions & 6 \\
coughing & 1 \\
\hline
\end{tabular}

Clinical manifestations include fever (7 cases, 63.6\%), gastrointestinal symptoms ( 9 cases, $81.8 \%$ ) and drowsiness (5 cases, 45.5\%), seizures (6 cases, 54.5\%), dizziness (2 cases, 18.2\%), neck resistance $(+)$ (1 case, 9.1\%), neck resistance suspicious (3 patients, $27.3 \%$ ).

Serum mycoplasma antibody IgG was positive in 6 cases, 2 children with acute diarrhea and rotavirus positive. The appearance of cerebrospinal fluid, total white blood cell count, white blood cell classification, protein, glucose and chloride are all normal. In the electroencephalography examination, 9 cases were normal, and 2 cases showed diffuse slow waves. The clinical symptoms disappeared after 11 children were treated with anti-infectives, vitamin B and fluids to meet physiological needs, etc.

\subsection{Follow-up}

All the 11 cases showed mild clinical symptoms. The duration of the disease ranged from 8 days to 21 days. The prognosis of all cases was good. No further symptoms of fever, vomiting, diarrhea, lethargy or convulsion were reported during the follow-up period of 6 to 10 months.

\subsection{Characteristics of MRI}

All the 11 patients showed that isolated SCC lesions showed a slightly longer T1 signal and a slightly longer T2 signal, while the DWI showed high signal, and the ADC diagram showed low signal (Figure 1-4). After treatment, the lesion of the head MR was disappeared (Figure 5-6). In addition to SCC lesions, there were no other lesions in the skull. The location and morphology of the lesion in the corpus callosum: 4 cases were ovoid and located in the middle, 6 cases were banded and located in the middle, and 1 case was banded and dispersed.

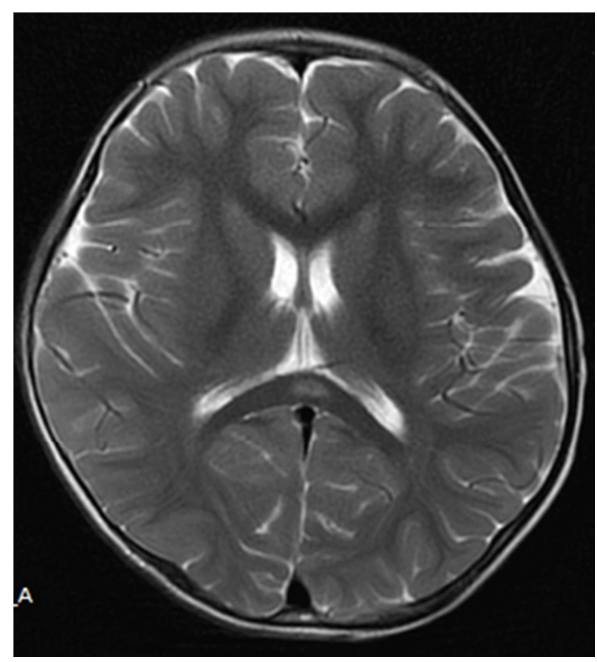

Figure 1. Axial position on T2WI The center of the pressure of the corpus callosum has a slightly higher signal of a band.

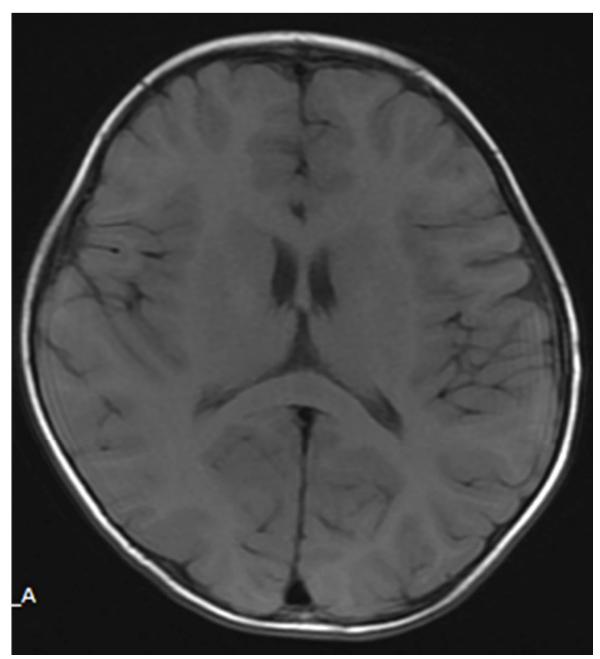

Figure 2. Axial T1WI The positive lesion of the pressure part of the corpus callosum presents a slightly lower signal. 


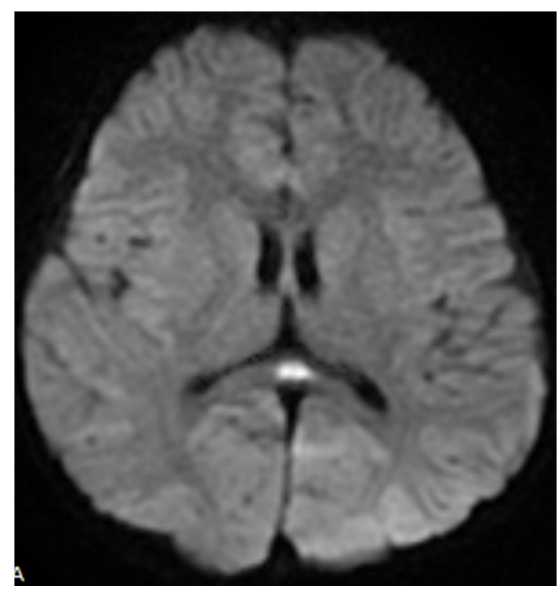

Figure 3. DWI $(b=1000)$ The positive lesion in the pressure part of the corpus callosum is a high signal.

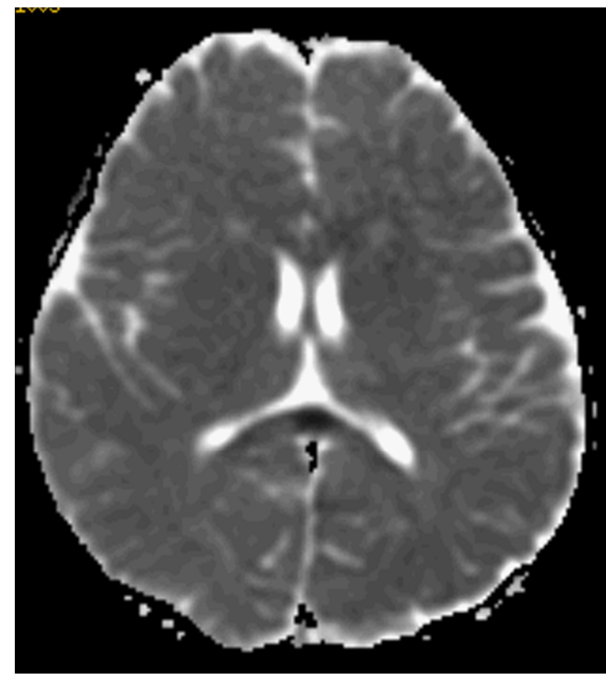

Figure 4. ADC map The positive lesion of the pressure part of the corpus callosum is a low signal.

Figure 1-4: Female, 2 years old. Chief complaint: vomiting 2 days, convulsions 1 times. After admission, she was given anti-infective drugs, mannitol, and vitamin B for 2 days, and then MRI was performed.

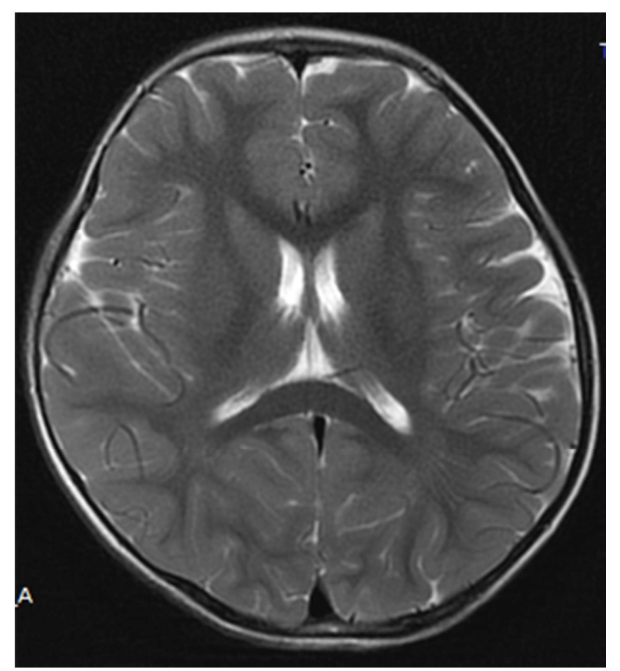

Figure 5. Axial T2WI The lesion of the corpus callosum disappeared.

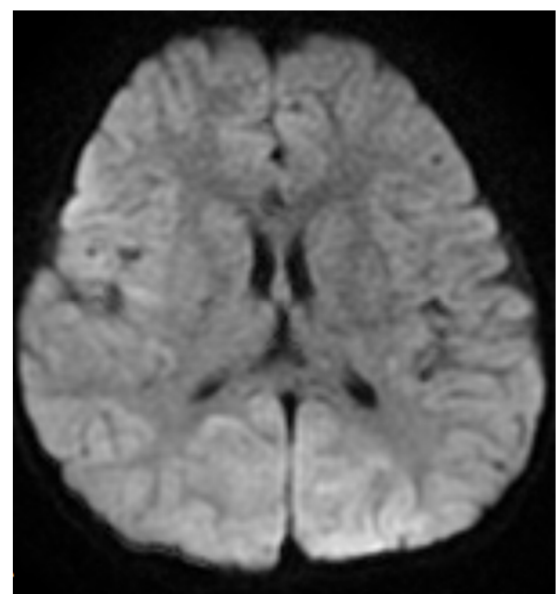

Figure 6. DWI $(b=1000)$ The lesion of the corpus callosum disappeared.

Figure 5-6: In the same patient, MRI images were reviewed 29 days after onset.

\section{Discuss}

\subsection{Etiological Analysis}

Most patients have virus infection, such as influenza virus, mumps virus, measles virus, adenovirus, rotavirus and so on [2-8]. Of the 11 patients, 5 were associated with respiratory tract infection and 2 were positive with rotavirus. Cerebrospinal fluid protein, glucose and chloride were normal, mycobacterium tuberculosis DNA, mycoplasma pneumoniae DNA, herpes simplex virus universal DNA and EBV-DNA were all negative. According to a statistical result, 3.3 percent of MERS is caused by bacteria, such as escherichia coli and salmonella enteritis [5]. Recently, a Japanese scholar reported 1 case of light encephalitis caused by urinary tract infection, and klebsiella was found in the urine test [9]. Except infection, the use of antiepileptic drugs and drug withdrawal are another major reason [10]. In an academic literature, epilepsy patients were divided into two groups, epilepsy with reversible SCC lesions and epilepsy without such lesions. There was no statistical difference in the level of antiepileptic drugs in the two groups [11].

\subsection{Pathogenesis}

The pathogenesis of MERS is not yet known and is thought to be a cytotoxic edema. It may cause the release of a series of inflammatory cytokines for pathogens or antibodies, leading to edema in the myelin sheath of the lesion $[10,12$, 13].

MRI is the best neuroimaging technique, especially the diffusion weighted imaging (DWI) sequence, which is sensitive to the detection of white matter lesions. In neonatal ischemic encephalopathy, hypoglycemic encephalopathy or acute disseminated encephalomyelitis, DWI can detect cytotoxic edema or angiogenic edema sensitively. Therefore, in MRI examination of brain, DWI is necessary and helpful to analyze the nature of the lesion.

In the MRI images of the cases in this paper, the lesions 
showed a high signal in T2WI and DWI sequences, and T1WI showed slightly lower signal, and the ADC diagram showed a low signal, confirming that this callosum pressure lesion was a cytotoxic edema. Kerstin et al. and Olga et al. had used diffusion tensor imaging (DTI) to detect epilepsy patients, and the results showed that SCC had cytotoxic edema (myelin edema of glial cells). Since the nerve fibers are not destroyed, the lesion is reversible [10, 13].

Some foreign scholars, after studying the composition of the white matter fibers in the corpus callosum, indicated that the pressure part of the corpus callosum lacked an epinephrine receptor. This can lead to the deficiency of the self-regulating mechanism, especially in the condition of hypoxia or continuous high perfusion, and thus the lesion of the site [14]. Someone used magnetic resonance imaging (MRS) technique to detect a case of staphylococcal meningitis with reversible SCC lesions. MRS showed the increase of lactic acid peak and inositol peak in the lesion area of the corpus callosum. This means that there is an increase in anaerobic glycolysis in the lesion and hyperosmosis in the lesion [15], thereby confirming that the callosum pressure is susceptible to damage.

\subsection{Clinical Manifestations, Treatment and Follow-up}

Most of the damage of the callosal pressure is visual impairment, and more common with homonymous hemianopia. Sometimes there are symptoms such as weakness and aphasia. In rare cases, only headache and dizziness can occur. However, the clinical manifestation is relatively hidden and the prognosis is good. On the other hand, there are few domestic studies on children's MERS, and children's clinical manifestations are different from adults. The symptoms are atypical and may be related to the immature corpus callosum development. In cases of this article, the most common clinical manifestations were fever, gastrointestinal symptoms (vomiting, diarrhea), lethargy and convulsions. Only 2 children experienced dizziness, but their previous history also had an unknown reason for the dizziness, which appeared to be less relevant to the disease. The course of this type of lesion is within 1 month [6], which can be treated with anti-virus and symptomatic treatment. After the clinical symptoms improve, the lesion disappears. All of the 11 cases, confirmed by brain MRI, were treated with antivirus and symptomatic treatment. The MRI of the brain was reviewed within 1 month, indicating that the lesion of the corpus callosum was completely disappeared, consistent with the relevant reports of the literature $[6,16]$.

\section{Conclusions}

In summary, the lesion of the reversible isolated corpus callosum is more common in children with mild encephalitis, and the most common clinical manifestations are fever, gastrointestinal symptoms, somnolence and convulsions. The appearance of cerebrospinal fluid, white blood cell count and classification, protein, glucose and chloride are all normal. After anti-infection and symptomatic treatment, the children can be cured within 1 month. This type of lesion is a reversible cytotoxic edema that can disappear after clinical symptoms improve. MRI examination can detect such lesions, which can prompt the children with good prognosis. After an early MRI examination, and a review of MRI after improvement of clinical symptoms, the doctor can get a clear diagnosis, and the DWI sequence is indispensable.

\section{Acknowledgements}

This project is funded from the basic research project of the Shanxi Science and Technology Department of China. Project number: 2015011100. Project name: Study on the pathogenesis of children isolated corpus callosum with mild encephalitis.

\section{References}

[1] Tada H, Takanashi J, Barkovich AJ, et al. Clinically mild encephalitis/encephalopathy with a reversible splenial lesion. Neurology 2004; 63:1854-58.

[2] Malhotra HS, Garg RK, Vidhate MR, et al. Clinical significance of transient lesion in splenium of corpus callosum. Ann Indian Acad Neurol 2012; 15:151-57.

[3] Bulakbasi N, Kocaoglu M, Tayfun C, et al. Transient splenial lesion of the corpus callosum in clinically mild influenza-associated encephalitis/encephalopathy. AJNR 2006; 27:1983-86.

[4] Ganapathy S, Ey EH, Wolfson BJ, et al. Transient isolated lesion of the splenium associated with clinically mild influenza encephalitis. Pediatr Radiol 2008; 38:1243-45.

[5] Hoshino A, Saitoh M, Oka A, et al. Epidemiology of acute encephalopathy in Japan, with emphasis on the association of viruses and syndromes. Brain Dev 2012; 34:337-43.

[6] Takanashi J. Two newly proposed infectious encephalitis/encephalopathy syndromes [J]. Brain Dev 2009; 31:521-28.

[7] Takanashi J, Shirai K, Sugawara Y, et al. Kawasaki disease complicated by mild encephalopathy with a reversible splenial lesion (MERS). J Neurol Sci 2012; 315:167-69.

[8] Matsuoka T, Yodoshi T, Sugai M, et al. A Case of Mild Encephalopathy with a Reversible Splenial Lesion Associated with G5P Rotavirus Infection. Case Rep Pediatr. 2013; 2013:197163.

[9] T Okamoto, Y Sato, T Yamazaki, A Hayashi. Clinically mild encephalitis/encephalopathy with a reversible splenial lesion associated with febrile urinary tract infection. European Journal of Pediatrics 2014; 173:533.

[10] K Anneken, S Evers, S Mohammadi, W Schwindt, M Deppe. Transient lesion in the splenium related to antiepileptic drug: case report and new pathophysiological insights. Seizure European Journal of Epilepsy 2008; 17:654-57.

[11] G Gröppel, P Gallmetzer, D Prayer, W Serles, C Baumgartner. Focal lesions in the splenium of the corpus callosum in patients with epilepsy. Epilepsia 2009; 50: 1354-60. 
[12] Fumihito N, Tomohiro K, Tomoko $\mathrm{M}$, et al. Reversible Splenic Lesion in a Patient with Febrile Infection-Related Epilepsy Syndrome (FIRES). Neuropediatrics 2013; 44:291-94.

[13] Olga P, Jacqueline D, Francois L, et al. Reversible Cytotoxic Edema in the Splenium of the Corpus Callosum Related to Antiepileptic Treatment: Report of Two Cases and Literature Review. Epilepsia 2005; 46:1633-36.

[14] Hackett PH, Yarnell PR, Hill R, et al. High-altitude cerebral edema evaluated with magnetic resonance imaging: clinical correlation and pathophysiology. JAMA 1998; 280:1920-25.

[15] Lin YW, Yu CY. Reversible focal splenium lesion-MRS study of a different etiology. Acta Neurol Taiwan 2009; 18:203-6.

[16] Qiong Fang, Lang Chen, Qiaobin Chen, Zhi Lin, et al. Clinically mild encephalitis/encephalopathy with a reversible splenial lesion of corpus callosum in Chinese children. Brain Dev, 2017; 39 (4):321-326. 